EPJ Web of Conferences 93,02019 (2015)

DOI: $10.1051 /$ epjconf/ 20159302019

(C) Owned by the authors, published by EDP Sciences, 2015

\title{
Current and Future Research at DANCE
}

\author{
M. Jandel ${ }^{1}$, a , B. Baramsai ${ }^{1}$, T. A. Bredeweg ${ }^{1}$, A. Couture ${ }^{1}$, A. Hayes ${ }^{1}$, T. Kawano ${ }^{1}$, S. Mosby ${ }^{1}$, G. Rusev ${ }^{1}$, I. Stetcu ${ }^{1}$, \\ T. N. Taddeucci ${ }^{1}$, P. Talou ${ }^{1}$, J. L. Ullmann ${ }^{1}$, C. L. Walker ${ }^{1}$, and J. B. Wilhelmy ${ }^{1}$ \\ ${ }^{1}$ Los Alamos National Laboratory, Los Alamos, 87545, New Mexico, USA
}

\begin{abstract}
An overview of the current experimental program on measurements of neutron capture and neutron induced fission at the Detector for $\underline{A}$ dvanced Neutron Capture Experiments (DANCE) is presented. Three major projects are currently under way: 1) high precision measurements of neutron capture cross sections on Uranium isotopes, 2) research aimed at studies of the short-lived actinide isomer production in neutron capture on ${ }^{235} \mathrm{U}$ and 3) measurements of correlated data of fission observables. New projects include developments of auxiliary detectors to improve the capability of DANCE. We are building a compact, segmented NEUtron detector Array at DANCE (NEUANCE), which will be installed in the central cavity of the DANCE array. It will provide experimental information on prompt fission neutrons in coincidence with the prompt fission gamma-rays measured by $160 \mathrm{BaF}_{2}$ crystals of DANCE. Unique correlated data will be obtained for neutron capture and neutron-induced fission using the DANCE-NEUANCE experimental set up in the future.
\end{abstract}

\section{Introduction}

Current research at DANCE is focused on many areas of basic and applied nuclear science. Data on neutron capture and neutron-induced fission, essential for many applications including nuclear energy, nuclear forensics and stockpile stewardship, were obtained in recent years at DANCE including the capture cross section of ${ }^{235} \mathrm{U}$ [1], ${ }^{239} \mathrm{Pu}[2],{ }^{237} \mathrm{~Np}$ [3], ${ }^{241} \mathrm{Am}$ [4] and ${ }^{238} \mathrm{U}$ [5]. The DANCE collaboration contributes significantly to improve our understanding of photon strength functions (PSF) as well as nuclear level densities (NLD) through dedicated measurements of neutron capture reactions on selected isotopes. Detailed studies of the capture gamma-ray cascades from compound nucleus yielded many results on spins and parities of neutron resonances and models of PSF and NLD [6-13].

Currently, three major experimental programs are under way at DANCE. The first project includes a five-year long measurement campaign of Uranium isotopes. The precision cross section measurements with the DANCE array have been improved significantly for the major actinides ${ }^{235} \mathrm{U}$ [1] and ${ }^{239} \mathrm{Pu}$ [2] in recent years. Techniques for ratio measurements were developed and proved to yield results up to $1 \mathrm{MeV}$ of incident neutron energy. New measurements are underway to use ratio techniques for threshold fissioning actinides $-{ }^{234} \mathrm{U},{ }^{236} \mathrm{U}$ and ${ }^{238} \mathrm{U}$. In addition, new experimental tools and mathematical methods will be developed for determination of the capture cross section. Special care will be paid to development of a Monte-Carlo scheme for the total uncertainties in the determination of DANCE cross sections. Finally, we will

\footnotetext{
a e-mail: mjandel@lanl.gov
}

develop methodologies for absolute capture cross section measurements.

The second project is a three year long project focused on the role of the short-lived actinide isomers in a high neutron flux environment. In particular, we are interested in quantifying the population of two isomeric states in ${ }^{236} \mathrm{U}$ that were found to have an unexpectedly large yield in neutron capture on ${ }^{235} \mathrm{U}$ for resonance neutrons [14].

The third project is focused on the measurements of correlated data from neutron-induced and spontaneous fission. The prompt-fission gamma-ray (PFG) emission properties were measured at DANCE in recent years and fully correlated data on PFG energies and multiplicities were obtained for many actinides [15-17]. We have started studying a complicated de-excitation path following neutron-induced and spontaneous fission at DANCE using detailed models of fission fragment de-excitation [18-20] and also a semi-empirical model using parameterized correlated distributions $[16,17]$. In this project we will carry out measurements of fission fragment kinetic energies in coincidence with the PFG at DANCE.

The review of these three projects will be given in following sections.

\section{Nuclear data for basic and applied science}

A ratio method is widely used in the measurements of neutron-induced reaction cross sections. Typically, the measurements with the sample of interest and neutron flux are carried out at the same time. In this case, the so-called "neutron monitors" use a sample with a very well known neutron-induced reaction cross section, such as ${ }^{3} \mathrm{He},{ }^{10} \mathrm{~B}$, 


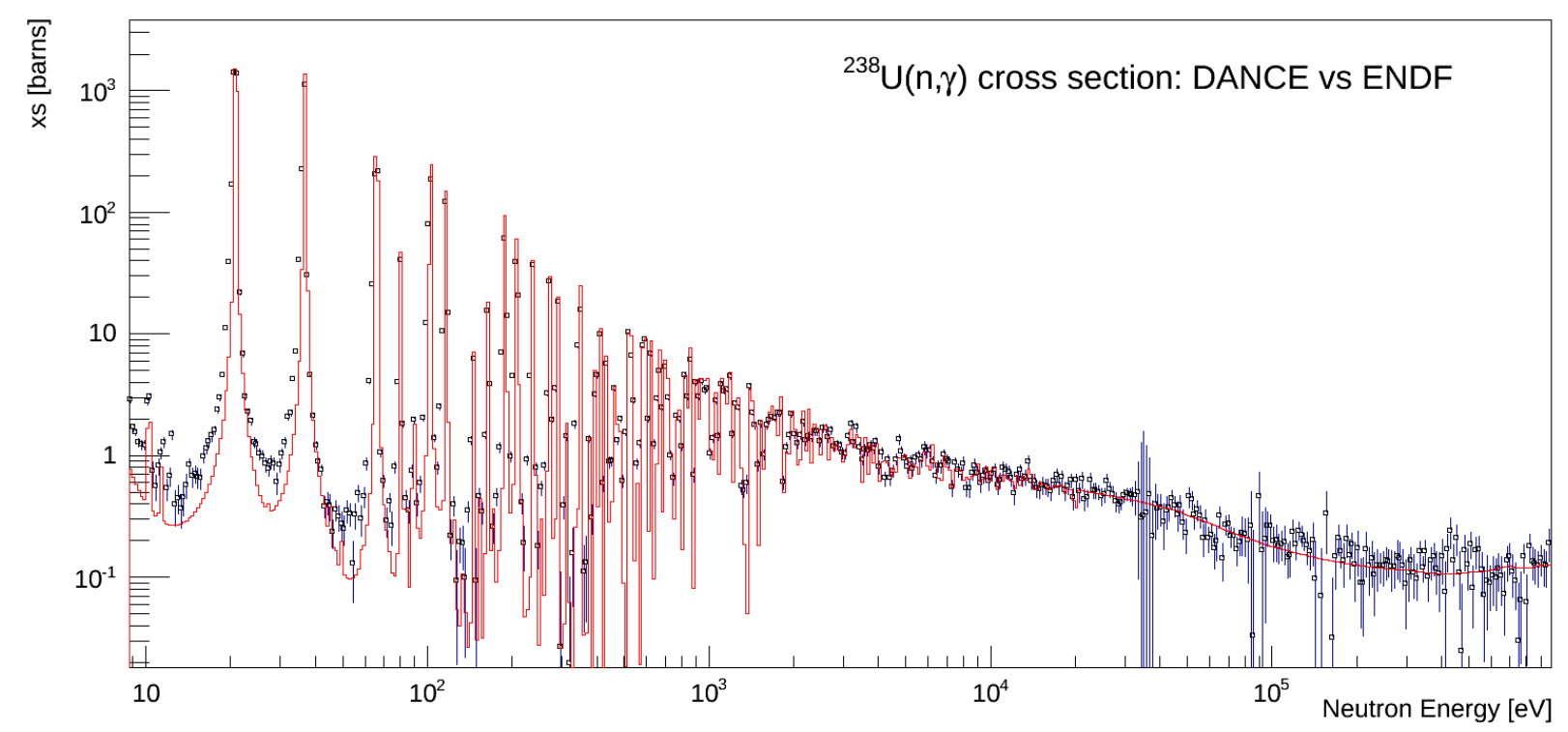

Figure 2. Capture cross section of ${ }^{238} \mathrm{U}$ obtained using a ratio of reaction rates ${ }^{238} \mathrm{U}(\mathrm{n}, \gamma) /{ }^{235} \mathrm{U}(\mathrm{n}, \mathrm{f})$ from two independent measurements [1] and [3]. Preliminary DANCE results (empty squares) are compared to ENDF-B/VII.0 evaluation (solid line).

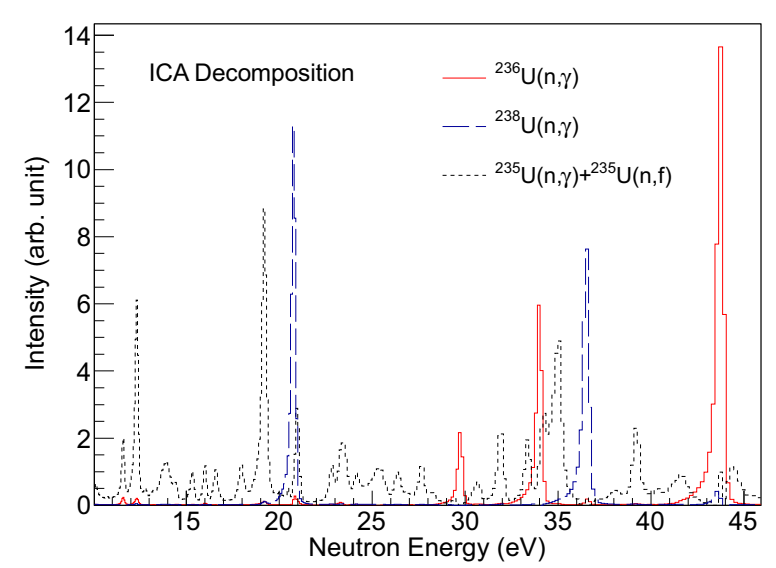

Figure 1. Separation of the different reaction rates using ICA method.

or ${ }^{6} \mathrm{Li}$, and measure the neutron flux in such manner that it does not perturb the measurement of the reaction rate of interest. Cross section measurements can also be carried out in two independent measurements, using samples with the same diameter and the same position in the neutron flux. The neutron flux is assumed to not change for the two measurements. However, these methods suffer from uncertainties in the neutron flux normalization, sample uniformity and neutron beam profile. In recent years, we have developed a methodology, where we take advantage ot the fact that in one sample, two neutron-induced reactions take place simultaneously. In the case of ${ }^{235} \mathrm{U}$ or ${ }^{239} \mathrm{Pu}$ samples, neutron capture and neutron-induced fission reactions occur with the rates proportional to their cross section at each incident neutron energy. We have shown [1, 2], that the neutron-induced fission reaction rates can be obtained with DANCE using the methods of gamma-ray calorimetry, where we used the tails of the prompt fission gammaray multiplicity and total energy distributions, which are much wider compared to these distributions from neutron capture and background. It is very convenient that the same number of sample atoms face the same neutron flux and the ratio of the two reactions completely removes the neutron flux from the analysis. However, for threshold fissioning actinides such as ${ }^{234} \mathrm{U},{ }^{236} \mathrm{U}$ and ${ }^{238} \mathrm{U}$, the neutron induced fission is suppressed by few orders of magnitude compared to neutron capture for incident neutron energies below $1 \mathrm{MeV}$. In this case, our approach is to prepare mixed targets, where ${ }^{235} \mathrm{U}$ will be mixed in at a given concentration. This way we can control the amount of neutron-induced fission from ${ }^{235} \mathrm{U}$ in the sample and also use its neutron-induced fission reaction rate as a neutron flux monitor reaction. The chemistry of Uranium is not dependent on isotopic number and therefore the $\mathrm{U}$ isotopes are uniformly distributed in the sample. One complication remains, and that is the contribution from the neutron capture on ${ }^{235} \mathrm{U}$ which needs to be removed.

Our first attempt to measure the capture cross section using a mixture target was not very successful. The mixture of ${ }^{236} \mathrm{U}$ and ${ }^{235} \mathrm{U}(80 / 20 \%)$ contained also a significant amount of ${ }^{238} \mathrm{U}$. This was revealed only after the measurement when a high precision mass spectrometry of the solution was performed. Too many components exist in our data, and it does not seem to be plausible to extract a cross section with small uncertainties from such data. We have tried to use an independent component analysis (ICA) [21] method to separate reactions from the measured data. The method seems to be efficient in the resolved resonance region, as shown in Figure 1, though its applicability remains to be demonstrated in future. 
Preliminary results of the ${ }^{238} \mathrm{U}$ neutron capture cross section were obtained using a ratio of reaction rates ${ }^{238} \mathrm{U}(\mathrm{n}, \gamma)$ and ${ }^{235} \mathrm{U}(\mathrm{n}, \mathrm{f})$, measured in two independent measurements described in [3] and [1], respectively. In Figure 2, a relative capture cross section of ${ }^{238} \mathrm{U}$ (normalized in the resonance region between $10-100 \mathrm{eV}$ ) is shown for incident neutron energy between $10 \mathrm{eV}$ and $1 \mathrm{MeV}$. The results were obtained using the equation:

$$
\sigma_{n \gamma}^{r e l}\left({ }^{238} U\right) \propto \frac{N_{n \gamma}\left({ }^{238} U\right)-B}{N_{n f}\left({ }^{235} U\right)} \sigma_{n f},
$$

where the background rates $B$ were obtained using ${ }^{208} \mathrm{~Pb}$ sample measurements described in Ref. [1]. Further work will include studies of scattering and self-shielding corrections to validate the ratio approach for independent measurements. The advantage of using the ${ }^{235} \mathrm{U}$ neutroninduced fission reaction is that we can identify this reaction without any background contributions up to $1 \mathrm{MeV}$ of incident neutron energy. Using only prompt fission gamma rays to identify this reaction removes the need for fission fragment detectors and allows us to use thick samples of ${ }^{235} \mathrm{U}$ and therefore reduce statistical uncertainties significantly.

\section{Short-lived actinide isotope production after neutron capture}

The preliminary studies of capture gamma-ray cascades after neutron capture of ${ }^{235} \mathrm{U}$ revealed an unexpectedly large population of isomeric states in the ${ }^{236} \mathrm{U}$ compound nucleus [14]. At incident neutron energy of $8 \mathrm{eV}$, almost $20-30 \%$ of the capture cascades pass through one of the two isomeric states - one at $1 \mathrm{MeV}\left(t_{1 / 2}=100 \mathrm{~ns}\right)$ and one at $680 \mathrm{keV}\left(t_{1 / 2}=3.7 \mathrm{~ns}\right)$ of excitation energy. The decay scheme depicting the population of the $1 \mathrm{MeV}$ isomer with the delayed emission of gamma-rays to the ground state of ${ }^{236} \mathrm{U}$ is shown in Figure 3. The production of these isomers may become important in a high neutron flux environment where the isomeric state lives long enough to capture a second neutron. In the case of ${ }^{236} \mathrm{U}$ isomers, the neutroninduced fission cross section below $1 \mathrm{MeV}$ is expected to be 2 orders of magnitude higher than the cross section for the ground state of ${ }^{236} \mathrm{U}$. A dedicated research and development started in 2013 to confirm the preliminary findings and quantify the yields of the ${ }^{236} \mathrm{U}$ isomers for different incident neutron energies. The most important development for this project is the design and construction of the new neutron detector array - NEUANCE. Using a liquid scintillator EJ-309, the detection of fission neutrons will be enabled using a pulse shape discrimination (PSD) technique. A unique trigger will be obtained for the neutron-induced fission reaction. In this manner, measurements with thick foils will be possible. In the past, we were able to trigger the fission reaction only with the use of single thin foil measurements with PPAC detectors $[14,15]$. In these measurements, the thickness of the actinide foils was limited to a few hundred $\mu \mathrm{g} / \mathrm{cm}^{2}$, and the counting statistics of these measurements were rather limited. With the current approach and NEUANCE in place, we will be able to

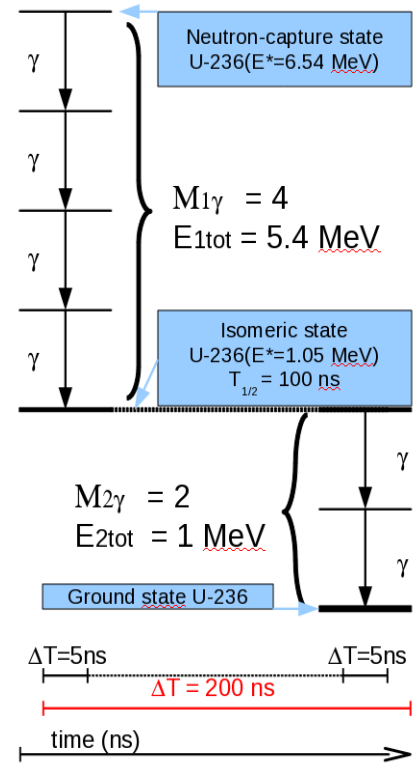

Figure 3. Schematics of the gamma-ray de-excitation from the neutron-capture state at $6.54 \mathrm{MeV}$ in the ${ }^{236} \mathrm{U}$ compound nucleus down to isomeric states. On the x-axis we show the time of the gamma-ray emission. The last two gamma rays at the end of the cascades are shown to be emitted at a later time because of the isomeric nature of the state at $1.05 \mathrm{MeV}$.

increase the counting statistics of prompt-fission and capture gamma-rays by two orders of magnitude, compared to previous measurements, over a wider range of incident neutron energies.

Geant4 simulations of NEUANCE have been developed for two possible geometrical configurations. Calculations are underway in order to estimate the efficiency of neutron detection, signal pileup, and the attenuation of gamma rays for different design configurations. Cascades of prompt fission neutrons and gamma rays are produced by the CGMF code $[19,20]$ and simulated further in Geant4. The modules that can simulate the scintillation light response (such as pulse shape, detection efficiency, etc.) are under development. A planned removal of the ${ }^{6} \mathrm{LiH}$ shell, currently installed in the center of the DANCE array, will introduce scattered-neutron background. In order to mitigate this background, we are designing new shielding options using a ${ }^{6} \mathrm{Li}$ and/or Boron loaded polyethylene.

A Geant4 model of the cylindrical NEUANCE detector that consists of 12 segments of liquid scintillators is shown in Figure 4. The length of each segment is $10 \mathrm{~cm}$ and the array covers almost the full solid angle around the target. The Geant 4 model of the NEUANCE detector is shown together with 80 crystals of DANCE using realistic dimensions and materials. The inner and outer radius of each segment of the NEUANCE detector was assumed to be 3 and $8 \mathrm{~cm}$, respectively. Prompt fission neutrons and gamma rays were simulated using the code CGMF $[19,20]$. We recorded hits in NEUANCE segments depending on the interaction type - proton recoils NP (corresponding to neutron inelastic scattering) above $100 \mathrm{keVee}$ (electron equivalent energy) and gamma ray events (elec- 


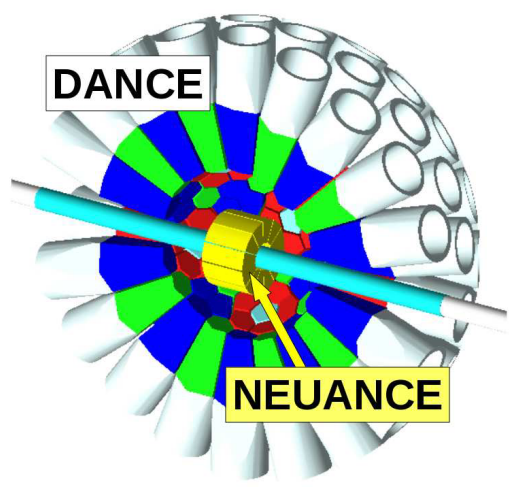

Figure 4. Geant4 simulation of NEUANCE: a twelve-segment liquid scintillator neutron detector in the center of DANCE.
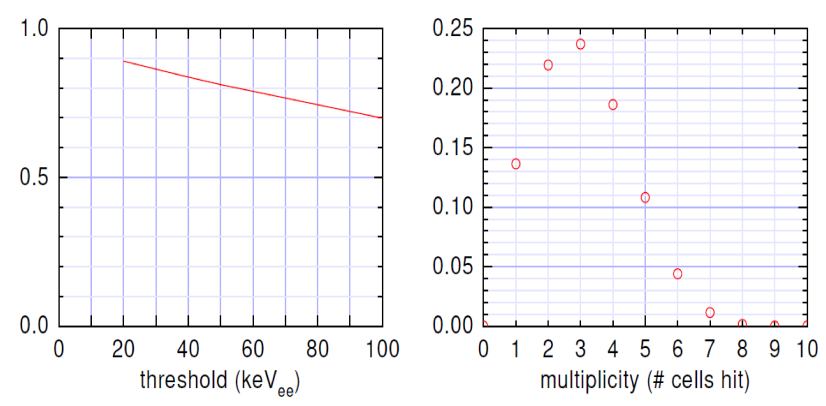

Figure 5. MCNP/Polimi calculations of the neutron detection efficiency for the ${ }^{252} \mathrm{Cf}$ source. Left) Fission trigger efficiency. Right) The number of NEAUNCE segments hit per fission.

tron deposition). For these events, the efficiency to detect at least one neutron in the event was very high: $52 \%$ and $42 \%$, for $E_{t h}>50 \mathrm{keVee}$ and $E_{t h}>100 \mathrm{keVee}$ detection thresholds, respectively. Alternatively, other designs of NEUANCE are under consideration. The different configurations will be tested off-line with a ${ }^{252} \mathrm{Cf}$ source, so the efficiency for fission neutron detection can be determined beforehand.

Similar simulations were performed using the MCNPX/Polimi code for the ${ }^{252} \mathrm{Cf}$ neutron source and cylindrical design of NEUANCE with 12 cells. The results are shown in Figure 4 where in the left panel we present the results of the detection efficiency of fission - detecting at least 1 neutron above the given threshold, shown on the $\mathrm{x}-$ axis. With the $100 \mathrm{keVee}$ threshold, an efficiency of $70 \%$ is expected to detect at least one fission neutron.

Other major developments are under way with the support of the Los Alamos National Laboratory Directed Research (LDRD) funding. A new data acquisition system is going to be implemented in the year 2014-2015. A purchase was made for new digitizers with 14-bit resolution, $500 \mathrm{MSample} / \mathrm{s}$ sampling rate, and FPGA on-board processing to allow for deadtimeless data acquisition from the $\mathrm{BaF}_{2}$ detectors of the DANCE array and NEUANCE. The FPGA signal processing flexibility allows us to incorporate different types of detectors, such as $\mathrm{Si}$, HPGe or $\mathrm{LaBr}_{3}$ detectors. We refer the reader to the paper by A. Couture

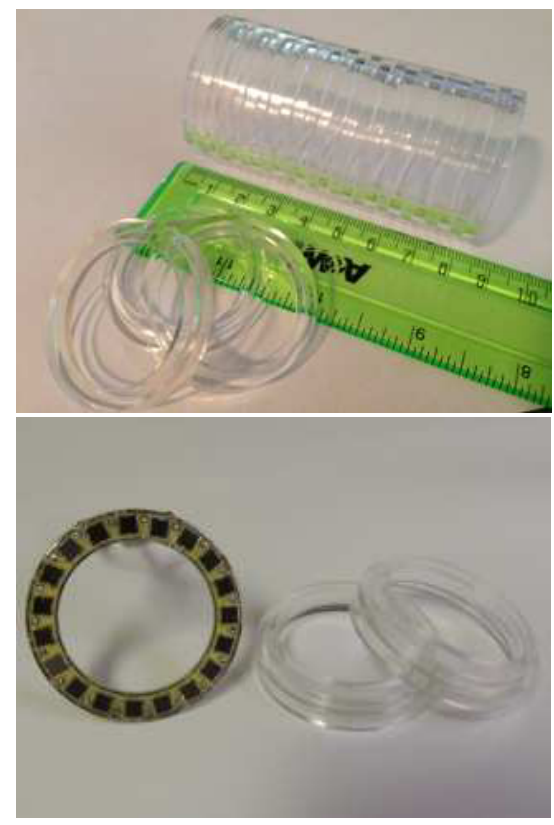

Figure 6. A prototype of the multifoil fission fragment tagging detector based on thin scintillator foils. Top panel) Acrylic light guide rings. Bottom panel) A ring of silicon photomultiplier sensors that will convert the light to electrical signals.

in these proceedings, for more detailed information on the new DANCE data acquisition development.

Work continues also on the development of new fission fragment detectors which will be used for the measurements of ${ }^{235} \mathrm{U}$ neutron-induced reactions within this project. Figure 6 shows a multi-foil design of the detector based on a thin scintillator foils (TSF), currently designed to hold 10 foils. The samples of ${ }^{235} \mathrm{U}$ will be deposited directly in the center of each TSF and will be placed in between the acrylic rings serving both as a TSF holder and the light guide (shown in the top panel of Figure 6). The fission fragments will produce light in the TSFs and the photons will be converted to electric signals at both ends by silicon photo-multipliers populated on the the PCB ring as shown in the bottom panel of Figure 6 .

\section{Correlated data on fission observables}

Prompt gamma-ray multiplicity distributions are critical to the modeling of fission reactions. Because neutrons carry off energy while gamma-rays carry off angular momentum, gamma-ray multiplicity distributions can give information about the initial distribution of angular momentum of the pre-emission fragments. The Los Alamos model assumes a simple triangle-shaped initial nuclear temperature distribution for fragments, and neutrons are evaporated according to a Weisskopf spectrum. This model is limited in the amount of detail it can provide; for instance, it predicts only an average number of neutrons emitted per fission. Newer approaches employ instead a Monte Carlo HauserFeshbach treatment, but they require more input parameters. Measurements of prompt gammas and neutrons can 


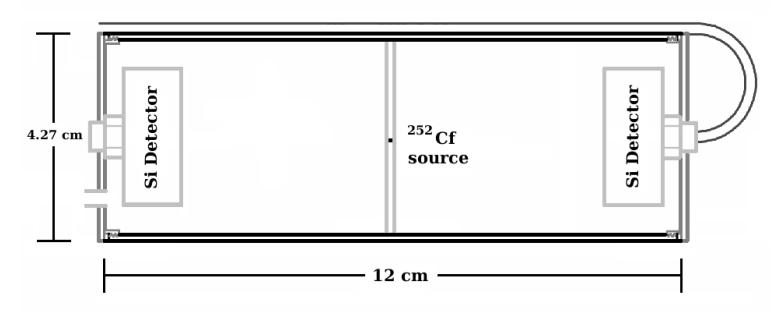

Figure 7. A design drawing of the container that holds two $\mathrm{Si}$ detectors and the ${ }^{252} \mathrm{Cf}$ source for the DANCE measurements of the total kinetic energy of fission fragments in coincidence with prompt fission gamma rays.

thus help constrain these models by providing information about pre-emission fragments which may otherwise be difficult to measure.

The ultimate goal of our experiment is to measure prompt gamma-ray energy and multiplicity distributions of neutron induced fission in ${ }^{235} \mathrm{U}$, simultaneously with the mass and kinetic energy of correlated fission fragments. We have constructed a fission fragment detector for use inside of the DANCE gamma-ray calorimeter. Initially, we plan to carry out measurements of total kinetic energy (TKE) of fission fragments emitted in the spontaneous fission of ${ }^{252} \mathrm{Cf}$. A simple detector setup using two Si detectors was designed for this purpose - see Figure 7. PFG properties will be measured in coincidence with fission fragments' TKE for the first time. In 2015, we plan to extend our measurements to neutron-induced fission of ${ }^{235} \mathrm{U}$.

\section{Summary}

Many developments are in progress to improve the capabilities of the DANCE detector array. New methodologies to measure relative and absolute cross sections are currently underway. The ${ }^{235} \mathrm{U}$ neutron-induced fission reaction is a candidate reference reaction for future measurements of capture cross section and was shown to work well in case of the ${ }^{238} \mathrm{U}$ capture cross section. Analysis of the systematic errors stemming from the data reduction, scattering corrections, and self-shielding is underway.

New data acquisition, the NEUANCE detector array, and fission fragment detectors will enable us to improve the accuracy and precision of new nuclear data. Most importantly, new capabilities developed upon completion of the three research projects described in this paper will prepare the ground for completely new measurements of correlated data, both in neutron capture and neutron-induced fission.

\section{Acknowledgements}

Work described in Section 2 was supported by the U.S. Department of Energy (DOE), Office of Science, Nuclear Physics under the Early Career Award \#LANL20135009. We gratefully acknowledge the support of the U.S. Department of Energy through the LANL/LDRD Program for the work described in Section 3. The first measurements of correlated data on fission observables, as described in Section 4 are supported by the Office of Defense Nuclear Nonproliferation Research and Development, US Department of Energy, NNSA. This material benefited from the use of the Los Alamos Neutron Science Center and is based upon work supported by the U.S. Department of Energy, National Nuclear Security Administration under Contract Number DE-AC52-06NA25396.

\section{References}

[1] M. Jandel et al., Phys. Rev. Lett. 109, 202506 (2012)

[2] S. Mosby et al., Phys. Rev. C 89, 034610 (2014)

[3] E.-I. Esch, et al., Phys. Rev. C 77, 034309 (2008)

[4] M. Jandel et al., Phys. Rev. C 78, 034609 (2008)

[5] J. L. Ullmann et al., Phys. Rev. C 87, 044607 (2013)

[6] S. A. Sheets et al., Phys. Rev. C 79, 024301 (2009)

[7] S. A. Sheets et al., Phys. Rev. C 76, 064317 (2007)

[8] A. Chyzh et al., Phys. Rev. C 84, 014306 (2011)

[9] B. Baramsai et al., Phys. Rev. C 85, 024622, (2012)

[10] B. Baramsai et al., Phys. Rev. C 87, 044609 (2013)

[11] G. Rusev et al., Phys. Rev. C 87, 054603 (2013)

[12] J. Kroll et al., Phys. Rev. C 88, 034317 (2013)

[13] G. Rusev et al., Phys. Rev. C 88, 057602 (2013)

[14] M. Jandel et al., in Proceeding of the 5th International Conference on "Fission and properties of neutron-rich nuclei" (ICFN5), Sanibel Island, November 4-10 (2012), World Scientific, p. 416

[15] J. L. Ullmann et al., Phys. Rev. C 87, 044607 (2013)

[16] M. Jandel et al., in Proceedings from GAMMA2 Workshop on Emission of Prompt Gamma-rays in Fission and Related Topics, Physics Procedia, submitted in 2014

[17] M. Jandel et al., in preparation for Phys. Rev C., 2015

[18] B. Becker, P. Talou, T. Kawano, Y. Danon, and I. Stetcu Phys. Rev. C 87,014617 (2013)

[19] I. Stetcu, T. Kawano, P. Talou, M. Jandel,Phys. Rev. C 88, 044603 (2013)

[20] I. Stetcu, T. Kawano, P. Talou, M. Jandel, Phys. Rev. C 90, 024617 (2014)

[21] Aapo Hyvarinen, IEEE Trans. on Neural Network, 10(3), 626 (1999) 
